\title{
Direct plasmonic detection of circulating $R A S$ mutated DNA in colorectal cancer patients
}

Roberta D'Agata ${ }^{1,4}$, Noemi Bellassai ${ }^{1}$, Matteo Allegretti ${ }^{2}$, Andrea Rozzi ${ }^{3,4}$, Saša Korom ${ }^{3,4}$, Alex Manicardi $^{3}$,\$, Elisa Melucci ${ }^{5}$, Edoardo Pescarmona ${ }^{5}$, Roberto Corradini ${ }^{3,4}$, Patrizio Giacomini $^{2}$, Giuseppe Spoto ${ }^{1,4^{*}}$

${ }^{1}$ Department of Chemical Sciences, University of Catania, Viale Andrea Doria 6, 95122, Catania, Italy.

${ }^{2}$ Oncogenomics and Epigenetics, IRCSS Regina Elena National Cancer Institute, Via Elio Chianesi, 53, 00144 Rome, Italy.

3 Department of Chemistry, Life Sciences and Environmental Sustainability, University of Parma, Parco Area Delle Scienze, 17/A, 43124, Parma, Italy.

${ }^{4}$ INBB, Istituto Nazionale di Biostrutture e Biosistemi, Viale delle Medaglie d'Oro, 305, 00136 Roma, Italy.

${ }^{5}$ Pathology, IRCSS Regina Elena National Cancer Institute, Via Elio Chianesi, 53, 00144 Rome, Italy.

*Corresponding author

Email: giuseppe.spoto@unict.it

Keywords: plasmonics, gold nanoparticles, liquid biopsy, colorectal cancer, RAS mutations.

$\$$ Present Address: Department of Organic and Macromolecular Chemistry, Ghent University,

Krijgslaan 281-S4, 9000 Gent, Belgium 


\begin{abstract}
Although not yet approved by regulatory bodies, $R A S$ mutations in the blood of colorectal cancer (CRC) patients are emerging as biomarkers of acquired resistance to EGFR therapy. Unfortunately, reliable assays granting fast, real-time monitoring of treatment response, capable of refining retrospective, tissue-based analysis, are still needed. Recently, several methods for detecting blood $R A S$ mutations have been proposed, generally relying on multi-step, PCR-based, time-consuming and cost-ineffective procedures. By exploiting a liquid biopsy approach, we developed an ultrasensitive nanoparticle-enhanced plasmonic method for detecting $R A S$ single nucleotide variants (SNVs) in the plasma of CRC patients. The PCR-free method we developed is based on an imaging platform and allows the direct detection of $\sim 1$ attomolar $R A S$ sequences in plasma with a sandwich hybridization assay using peptide nucleic acids probes. The assay involves a simple pre-analytical procedure that does not require the extraction of tumor DNA from plasma and detects it in volumes as low as $40 \mu \mathrm{L}$ of plasma, which is at least an order of magnitude smaller than that required by state of the art liquid biopsy technologies. The most prevalent $R A S$ SNVs are detected in DNA from tumor tissue with $100 \%$ sensitivity and $83.33 \%$ specificity. Spike-in experiments in human plasma further encouraged assay application on clinical specimens. Assay performances were then proven in plasma from CRC patients and healthy donors, demonstrating its promising avenue for cancer monitoring.
\end{abstract}




\section{Introduction}

Precision oncology requires repeated, longitudinal profiling of cancer biomarkers to monitor disease progression and response to therapy ${ }^{1}$. Although very informative, tissue biopsy is not the ideal approach to obtain this information, because it is invasive, expensive, cannot be repeated at will, and often fails to recapitulate tumor heterogeneity, i.e. changes occurring in either space (e.g. in different tumor regions and metastatic foci) or time (e.g. adaptive selection and genetic drift throughout disease course $)^{2}$. Liquid biopsy (LB) has been deployed in a variety of clinical settings to overcome these limitations. LB investigates tumor biomarkers in body fluids such as blood, urine, cerebrospinal fluid, and saliva, and is revolutionizing precision oncology, also offering new opportunities for early cancer diagnosis ${ }^{3}$. The molecular profiling of cell-free DNA (cfDNA) ${ }^{4}$, and circulating tumor DNA (ctDNA) ${ }^{5}$ released in the peripheral blood has shown great potential to improve the clinical management of cancer patients $6,7,8$.

A variety of next-generation sequencing $(\mathrm{NGS})^{9}$ and PCR-based approaches ${ }^{10}$ have been developed to analyze DNA fragments freely circulating in the blood of cancer patients ${ }^{11}$. Their routine application as standard clinical tools, however, is still hampered by several factors including suboptimal sensitivity, specificity, robustness, simplicity and flexibility, along with complex preanalytical procedures of blood processing. Analysis time and assay costs must also be factored because appropriate throughput and short turnaround times are essential for molecular diagnosis in rapidly progressing, fragile patients 9 .

As to targeted NGS, it provides considerable throughput (many genomic alterations are simultaneously detected), but this comes at the cost of complexity. Amplicon barcoding/tagging technology is mandatory for LB-grade NGS. Extensive data elaboration requires dedicated bioinformatic pipelines and manpower, has high operating costs, and limits of detection may differ for the different genomic variants in the panel ${ }^{12,13}$. As compared to NGS, PCR-based approaches, including digital PCR (dPCR), interrogate a more limited set of mutations at one time and have limited scalability, but have superior analytical sensitivity. However, they are prone to sample 
contamination $^{14}$ and may be biased by volume variability of partition sizes ${ }^{15}$. Whereas NGS is a comprehensive screening tool, single-plex PCR monitors defined tumor alterations, typically those alterations expected a priori to be associated with resistance to therapy. $R A S$ mutations, arising in colorectal cancer (CRC) patients treated with antibodies to Epidermal Growth Factor Receptor (EGFR) are a case in point. Differences notwithstanding, a common major limitation of both NGS and $\mathrm{dPCR}$ is that they require a multi-step enzymatic amplification/expansion of input DNA, which introduces specific constraints and drawbacks including cumbersome pre-analytical procedures to isolate and concentrate template DNA from biological fluids.

In this context, plasmonic detection based on surface plasmon resonance imaging (SPRI) holds promise as an alternative tool, complementary to both NGS and PCR, to detect circulating tumorrelated alterations. It combines multiplexed biosensing capabilities with highly sensitive nucleic acid detection and needs no PCR amplification and target labelling ${ }^{16}$. The flexible design of the SPRI assay configuration can also benefit from the use of peptide nucleic acid (PNA) probes that enhance sequence selectivity and offer superior performances for the detection of single nucleotide variants (SNVs) compared to oligonucleotide probes ${ }^{17}$. The implementation of sandwich assay configurations using gold nanoparticles (AuNPs) ${ }^{18}$ conjugated to an oligonucleotide has been proved to push the analytical sensitivity of the plasmonic platform down to attomolar concentrations of genome equivalent ${ }^{19}$, thus opening new perspectives for the application of LB in cancer patients.

Herein, we describe an innovative nanoparticle-enhanced SPRI (NESPRI) platform for the diagnosis and monitoring of CRC patients by LB (Fig. 1). NESPRI can detect mutated or wild-type $R A S$ sequences directly in the plasma of patients and healthy donors, respectively, with no prior extraction and PCR amplification of the targets. We developed an assay targeting 11 selected KRAS or NRAS SNVs, known to be associated with primary or acquired resistance to treatment with antibodies to EGFR. These are located in KRAS exon 2 and NRAS exons 2 and 3, that are recurrently mutated in human cancer ${ }^{20}$, specifically in CRC patients. 
NESPRI detection of oncogenic DNA achieved diagnostic sensitivity of $100 \%$ and specificity of $83.33 \%$. Then, we successfully performed spike-in experiments with unique $R A S$ mutated DNA in plasma from healthy donors. Here, the assay successfully discriminated between wild-type and attomolar concentrated mutated DNAs. Finally, the NESPRI assay was applied to clinical samples from CRC patients demonstrating full discrimination between KRAS mutated ctDNAs over wildtype cfDNAs from healthy volunteers. Overall, our method incorporates multiplexed detection, is PCR-free and, most importantly, drastically simplifies cumbersome, time-consuming, and errorprone pre-analytical procedures aimed at separating/concentrating the target nucleic acids from the complex human plasma matrix, thus providing strong evidence that plasmonics may be used for enhanced cancer diagnosis and prognosis. The method also operates with a volume of human plasma which is at least an order of magnitude smaller than that required by state of art LB technologies.
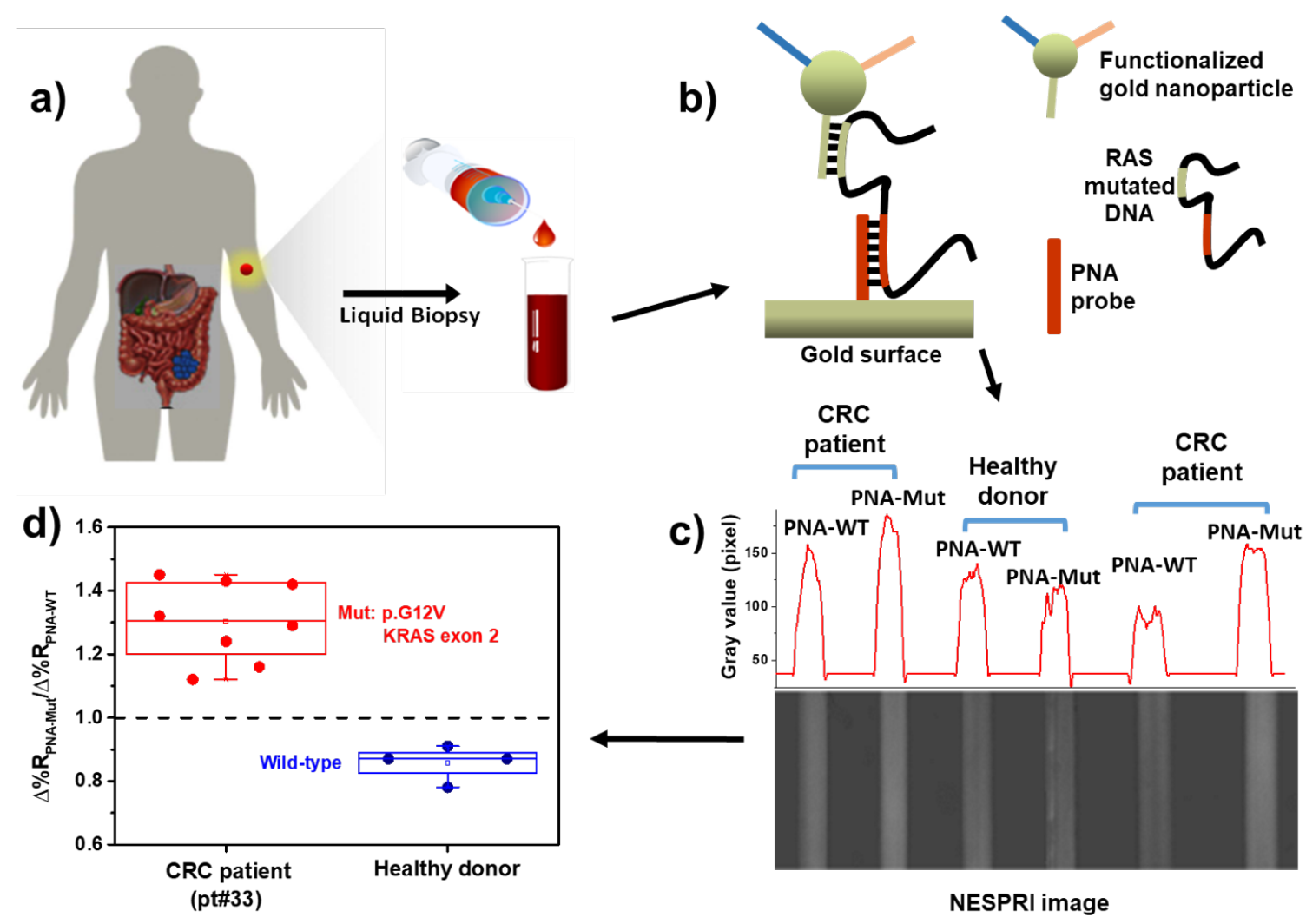

Fig. 1: Schematic overview depicting NESPRI method for liquid biopsy analyses: a) plasma from CRC patients is analyzed with no prior isolation and PCR amplification of the circulating target sequence; b) metal nanoparticle-based enhancement of the plasmonic signal is obtained using an optimized sandwich assay; c) 
the imaging setup allows multiplexed analysis. The plasma sample interacts with two PNA probes. d) The $R A S$ mutated DNA circulating in the blood of CRC patient and wild-type DNA of a healthy donor differently interact with PNA probes, thus producing different NESPRI responses.

\section{Results}

NESPRI detects $R A S$ mutated DNA from colorectal cancer tissue biopsies. Before LB optimization, we evaluated the NESPRI ability to detect $R A S$ mutations in tissue tumor DNA (tDNA) from surgically resected primary or metastatic CRC patients, e.g. in formalin-fixed paraffin-embedded (FFPE) samples. FFPE samples are routinely used as templates for clinical diagnostic $R A S$ profiling. Our tests were aimed at verifying assay performances with a defined tumor content and a less fragmented input compared to ctDNAs. To this end, tDNAs were extracted by conventional clinical-grade methods and analyzed with no prior amplification of the target sequence. Table 1 lists $R A S$ mutations selected among those that are most prevalent in CRC and other adenocarcinomas (e.g. lung and pancreas) ${ }^{8}$ and sequences of related PNA probes used to detect these mutations.

\begin{tabular}{|c|c|c|c|c|c|}
\hline Gene, exon & Sample ID & Mutation & PNA sequence* $\quad$ PN & acronym & $\mathbf{T} \mathbf{m}^{* * *}$ \\
\hline \multirow{7}{*}{ KRAS exon 2} & & Wild-type*** & CTACGCCACCAGCT & PNA-WTK2 & 73 \\
\hline & $\begin{array}{l}2604 \\
2914\end{array}$ & $\begin{array}{l}\text { p.G12D } \\
\text { p.G12D }\end{array}$ & CTACGCCATCAGCT & PNA-G12D & 70 \\
\hline & 2468 & p.G12C & CTACGCCACAAGCT & PNA-G12C & 73 \\
\hline & 2602 & p.G12V & CTACGCCAACAGCT & PNA-G12V & 73 \\
\hline & 2413 & p.G13D & CTACGTCACCAGCT & PNA-G13D & 70 \\
\hline & 2466 & p.G12R & CTACGCCACGAGCT & PNA-G12R & 75 \\
\hline & 2431 & p.G12S & CTACGCCACTAGCT & PNA-G12S & 69 \\
\hline \multirow{2}{*}{ NRAS exon 2} & & Wild-type*** & CCAACCACCACCAG & PNA-WTN2 & 73 \\
\hline & 2148 & p.G12D & CCAACCATCACCAG & PNA-NG12D & 70 \\
\hline \multirow{5}{*}{ NRAS exon 3} & & Wild-type*** & TACTCTTCTTGTCCAGCT & PNA-WTN3 & 70 \\
\hline & 1981 & p.Q61K & TACTCTTCTTTTCCAGCT & PNA-Q61K & 67 \\
\hline & 2582 & p.Q61H1 & TACTCTTCGTGTCCAGCT & PNA-Q61H1 & 74 \\
\hline & 2329 & p.Q61L & TACTCTTCTAGTCCAGCT & PNA-Q61L & 70 \\
\hline & 2335 & p.Q61R & TACTCTTCTCGTCCAGCT & PNA-Q61R & 72 \\
\hline
\end{tabular}

PNA probes were designed to have high melting temperatures (higher than $65^{\circ} \mathrm{C}{ }^{\circ} \mathrm{C}$ at $4 \mu \mathrm{M}$ strand concentration), as calculated according to an empirical model ${ }^{21}$; therefore, PNA probes of different 
lengths were selected to satisfy this criterion. The actual melting temperatures were measured after synthesis (Supplementary Table S4) and, while differing in some cases from those calculated, were found to be in the expected range $\left(68-83^{\circ} \mathrm{C}\right)$; the high melting temperatures denote high affinity for the complementary sequences, which is necessary for the ultrasensitive detection of the corresponding gene tracts; the site complementary to the mutated bases was placed in the internal part of the PNA sequence to maximize selectivity.

NESPRI assay we developed and tested is based on the sandwich detection approach depicted in Fig. 1b. PNA probes are first immobilized on the sensor surface; the target is then adsorbed on the surface, thus leading to the hybridization between the probe and the complementary sequence of the target. Appropriately functionalized AuNPs are used in the last step of the assay to enhance plasmonic detection. With this aim, AuNPs are decorated with oligonucleotides whose sequence is complementary to a portion of the sequence of the immobilized target not hybridized with the PNA probe.

NESPRI assay was aimed at detecting mutations in tDNA. For this reason, we performed the experiments immobilizing PNA probes $\left(0.1 \mu \mathrm{M}\right.$ in PBS, flow rate $10 \mu \mathrm{L} \mathrm{min}{ }^{-1}$. Immobilized probe density $3 \times 10^{-12}$ molecules $\mathrm{cm}^{-1}$ ) complementary to wild-type and mutated target sequences, respectively, on dithio-bis-succinimidyl propionate (DTSP)-modified SPRI gold chips. We obtained the spatial separation of the immobilized probes using a microfluidic device bearing parallel microchannels. Fig. 2 shows representative changes in per cent reflectivity $(\Delta \% \mathrm{R})$ over time detected for the immobilization of PNA-WTN2 and PNA-NG12D probes. The relevant SPR image (Fig. 2) highlights the achieved spatially separated immobilization of probes. 


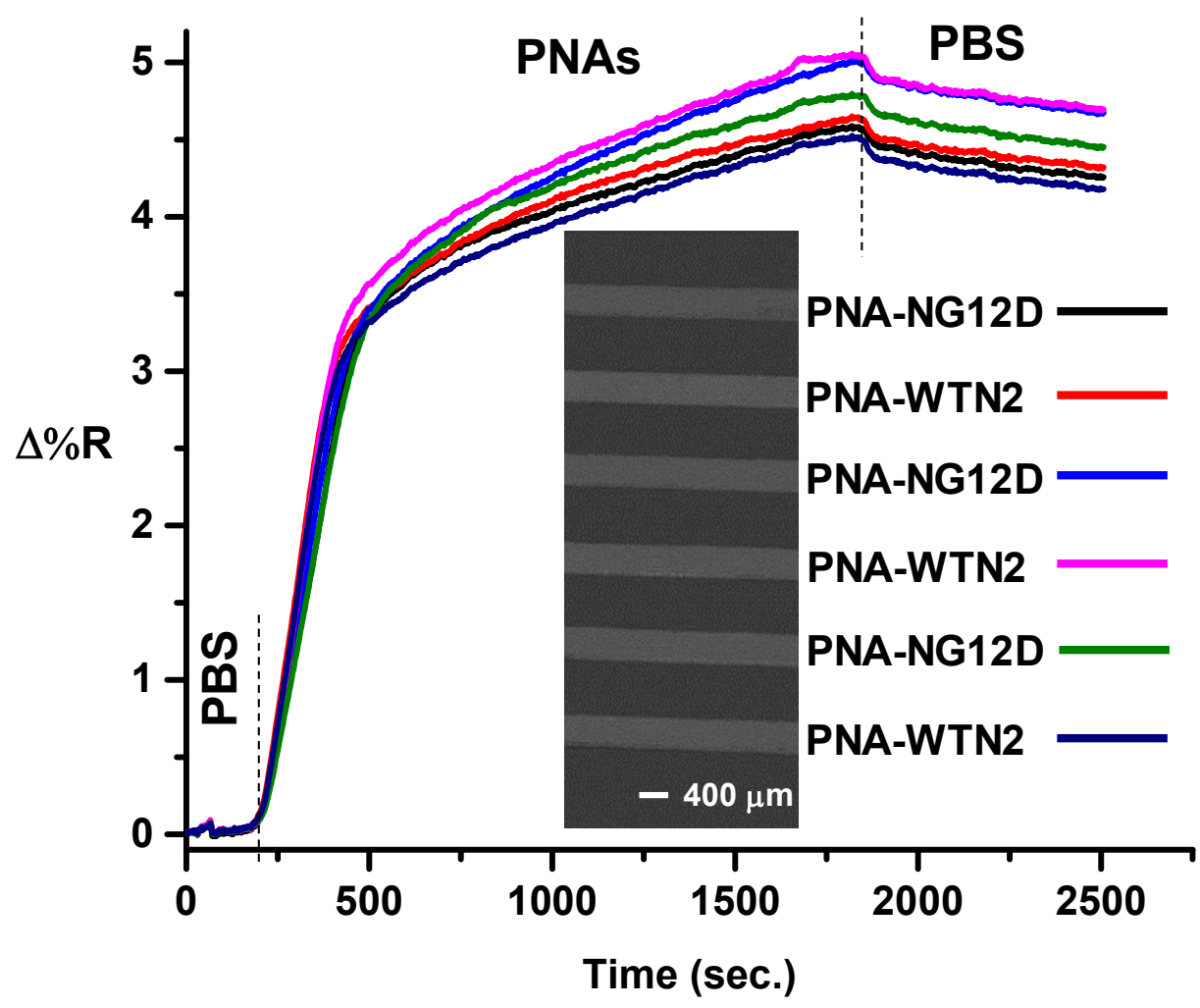

Fig. 2: Representative changes in per cent reflectivity $(\Delta \% \mathrm{R})$ over time detected for the immobilization of PNA-WTN2 and PNA-NG12D probes. An SPR image displaying the spatially confined immobilization of the probes (bright channels) is also shown.

Before NESPRI analysis, we fragmented tDNA by sonication and vortexing and denaturated it by heating at $95^{\circ} \mathrm{C}$. The sample was then cooled on ice to prevent strands reannealing and introduced in the microfluidic device to allow for the interaction with the immobilized PNA probes. The adsorption of tDNA on PNA probes complementary to wild-type and mutated sequences, respectively, was obtained introducing $300 \mu \mathrm{L}$ of a 5 pg $\mu \mathrm{L}^{-1}$ tDNA solution in two parallel microchannels (flow rate $10 \mu \mathrm{L} \mathrm{m^{-1 }}$ ). We detected SPRI signals close to the instrumental noise and not useful for samples discrimination during the adsorption (Supplementary Fig. S1). The surface was then washed with PBS for 15 minutes to reduce the amount of material non-specifically adsorbed on the surface of NESPRI sensor. 
As the last step of the assay, we used functionalized AuNPs. We were aimed at detecting $R A S$ mutations located in three exons; for this reason, we designed and optimized a suitable protocol to produce one single set of functionalized AuNPs (NPs@MixRAS) for NESPRI detection of all the selected $R A S$ mutations. In particular, we obtained NPs@MixRAS by conjugating AuNPs with a mix of three different oligonucleotides each complementary to an exposed DNA region of the exon carrying the mutations under test and the corresponding wild-type sequence. Such oligonucleotides were aimed at a region not involved in the hybridization with the relevant PNA probe (Fig. 1b). Table 2 shows the sequence of oligonucleotides we used to produce NPs@MixRAS and the exon targeted by each of them. NPs@MixRAS we used for the NESPRI detection showed a shelf-life of 14 months (Supplementary Table S6).

\begin{tabular}{|c|c|c|c|}
\hline Gene, exon & Mutation & Sequence & $\mathbf{T m}$ \\
\hline $\begin{array}{c}\text { KRAS exon } 2 \\
+ \\
\text { Wild-type }\end{array}$ & $\begin{array}{l}\text { G12A } \\
\text { G12D } \\
\text { G12C } \\
\text { G12V } \\
\text { G13D } \\
\text { G12R } \\
\text { G12S }\end{array}$ & 5' CAAGTTTATATTCAGTCAT 3' & $33.3^{\circ} \mathrm{C}$ \\
\hline $\begin{array}{c}\text { NRAS exon } 2 \\
+ \\
\text { Wild-type }\end{array}$ & G12D & 5' TTTCACACCAGCAAG 3' & $32.9^{\circ} \mathrm{C}$ \\
\hline $\begin{array}{c}\text { NRAS exon } 3 \\
+ \\
\text { Wild-type }\end{array}$ & $\begin{array}{c}\text { Q61K } \\
\text { Q61H1 } \\
\text { Q61L } \\
\text { Q61R }\end{array}$ & 5' TGTCCAACAAACAGG 3' & $33.5^{\circ} \mathrm{C}$ \\
\hline
\end{tabular}

After nanoparticle enhancement, our NESPRI assay was able to discriminate among $5 \mathrm{pg} \mu \mathrm{L}^{-1}$ solutions of wild-type and NRAS p.G12D mutated tDNAs. The nanoparticle enhancement highlighted the preferential interaction of wild-type tDNA with PNA-WTN2 (Fig. 3a, red line), whereas NRAS p.G12D tDNA preferentially interacts with PNA-NG12D probe (Fig. 3b, black line). To discriminate between wild-type and tDNA, we considered the ratio of NESPRI signals $(\Delta \% \mathrm{R})$ detected after the adsorption of NPs@MixRAS (Fig. 3) on surfaces resulting from the interaction of the selected sample with PNA probes complementary to the mutated (PNA-Mut) and 
wild-type (PNA-WT) sequences, respectively. We performed the experiments analyzing in parallel both the selected tDNA sample extracted from a tissue specimen of CRC patients and wild-type gDNA extracted from HT29 cells.

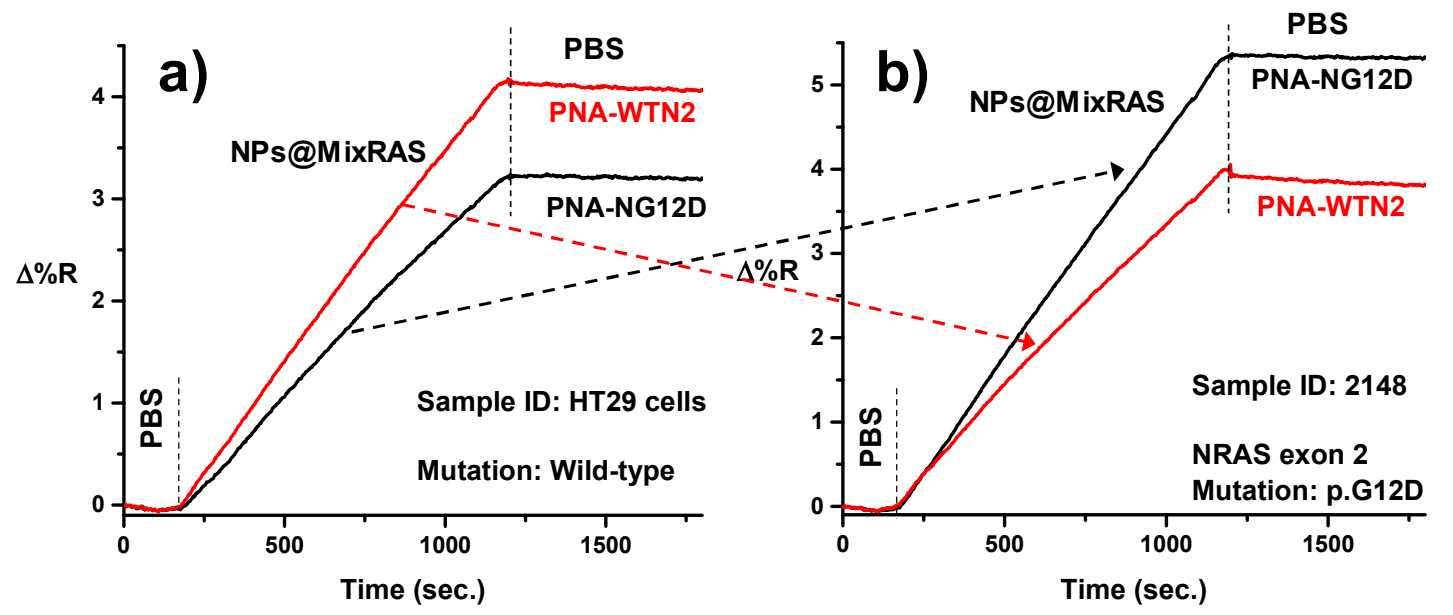

Fig. 3: $\Delta \%$ R over time for the adsorption of NPs@MixRAS (0.1 nM in PBS) on (a) wild-type (extracted from HT29 cells) and (b) p.G12D (NRAS) mutated tDNA previously adsorbed on surface-immobilized PNA-WTN2 (red line) and PNA-NG12D (black line) probes.

Fig. 4 and Supplementary Table S1 show results we got from the analysis of tDNA from CRC patients carrying $R A S$ mutations included among the most frequently occurring in one-third of human cancers. 


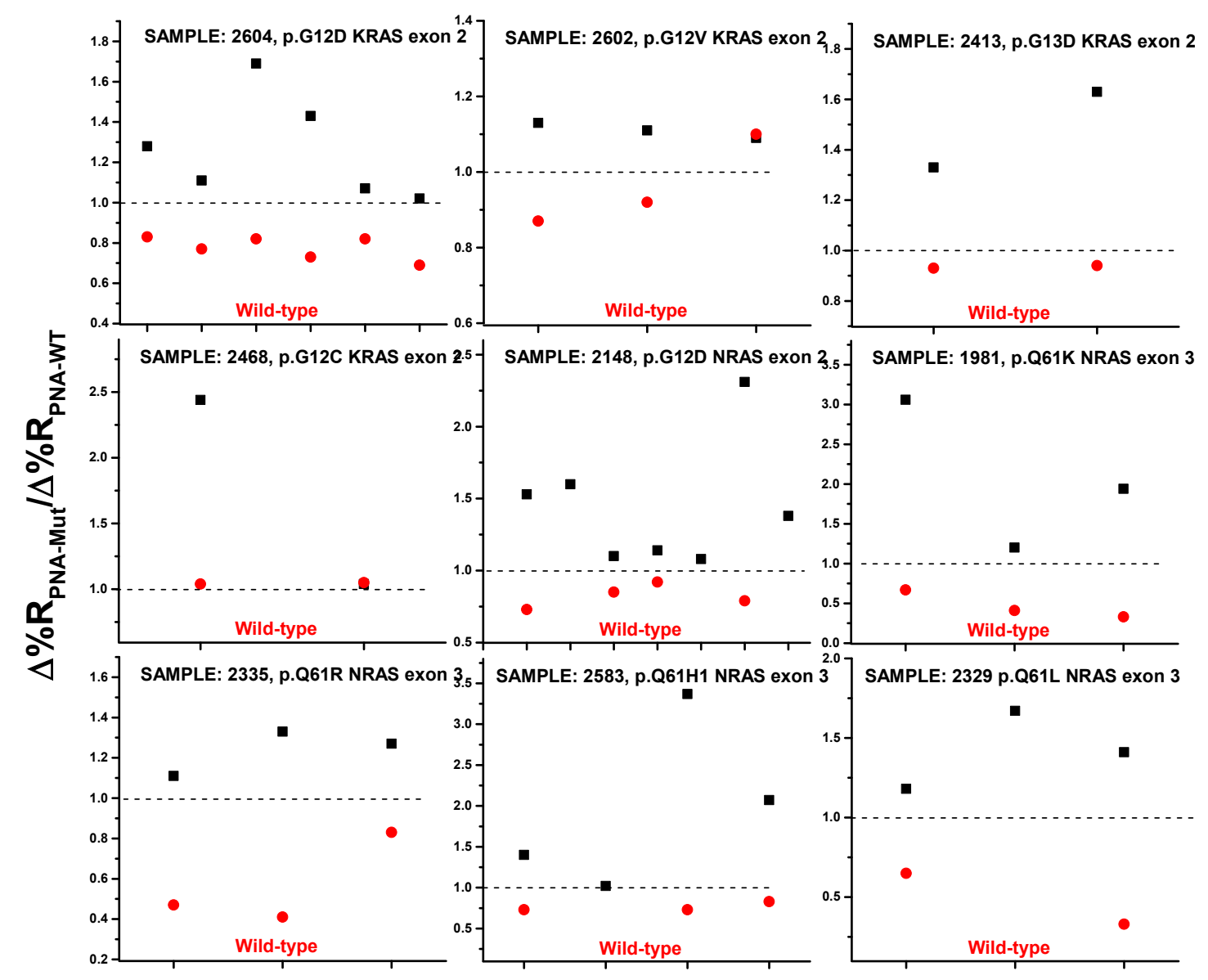

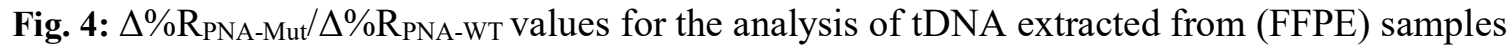
surgically resected from primary or metastatic CRC patients and wild-type gDNA.

According to $\Delta \% \mathrm{R}_{\text {PNA-Mut }} / \Delta \% \mathrm{R}_{\text {PNA-WT }}$ values, we constructed the receiver operating characteristic (ROC) curve plot (Fig. 5) to evaluate the diagnostic capability of the assay. NESPRI assay afforded AUC of 0.947 (95\% confidence interval 0.89-1.00, p-value $6.810^{-10}$. Table 3) supporting the high diagnostic capacity of the NESPRI method. 


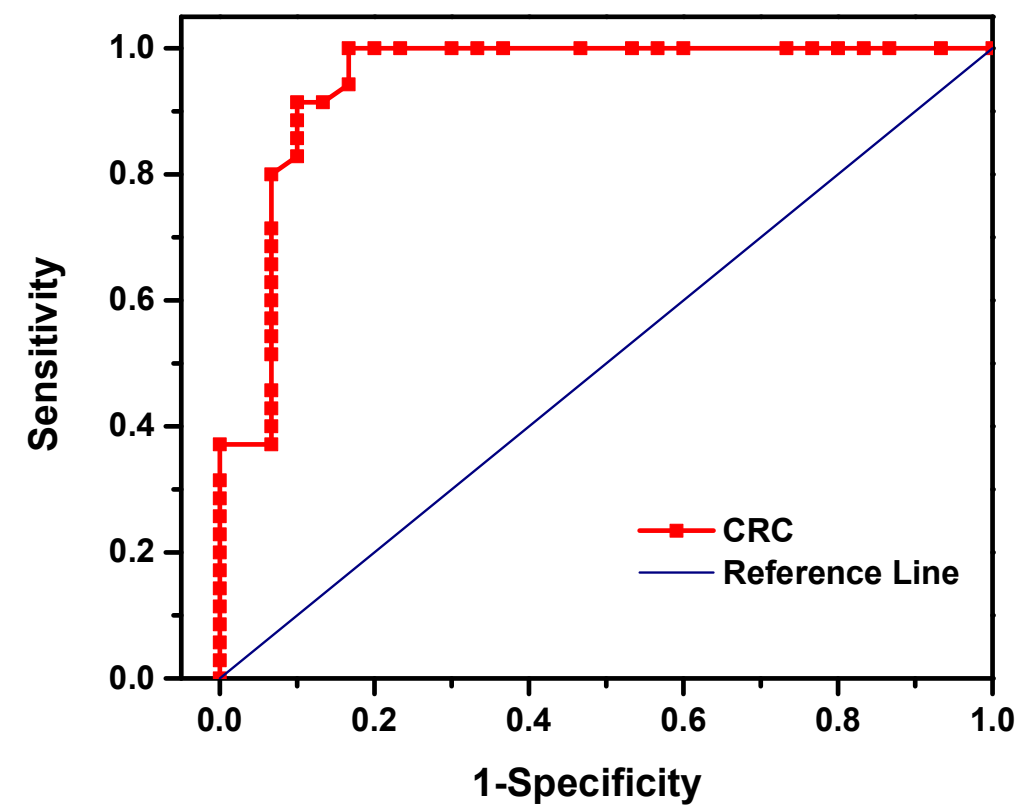

Fig. 5: Empirical receiver operating characteristic (ROC) curve of $\Delta \%$ RPNA-Mut/ $\Delta \%$ RPNA-WT ratio for NESPRI detection of $R A S$ mutations and wild-type gDNA.

NESPRI assay afforded a sensitivity of $100 \%$ and specificity of $83.33 \%$ based on the cutoff value of 0.98 for $\Delta \% \mathrm{R}_{\text {PNA-Mut }} \Delta \% \mathrm{R}_{\text {PNA-wT }}$ ratio (Table 3 ). The selected cutoff value is associated with the highest Youden index value (Youden index = sensitivity + specificity -1$)$.

\begin{tabular}{|lc|}
\hline \multicolumn{2}{|l|}{ Table 3. Sensitivity, specificity and AUC } \\
\hline Sensitivity & $100 \%$ \\
Specificitya & $83.33 \%$ \\
AUC & 0.947 \\
$95 \%$ confidence interval & $0.89-1.00$ \\
$p$-value & $6.810^{-10}$ \\
\hline $\begin{array}{l}{ }^{a} \text { Sensitivity and specificity were obtained at the } \\
\text { cutoff of } 0.98\left(\Delta \% \mathrm{R}_{\text {PNA-Mut }} / \Delta \% \mathrm{R}_{\mathrm{PNA}-\mathrm{WT}}\right) .\end{array}$ \\
\hline
\end{tabular}

We conducted additional experiments aimed at comparing NESPRI detection of tDNAs extracted from tissue biopsies of CRC patients with gDNA isolated from the peripheral blood mononuclear cells (PBMCs) of healthy donors instead of cell lines. 

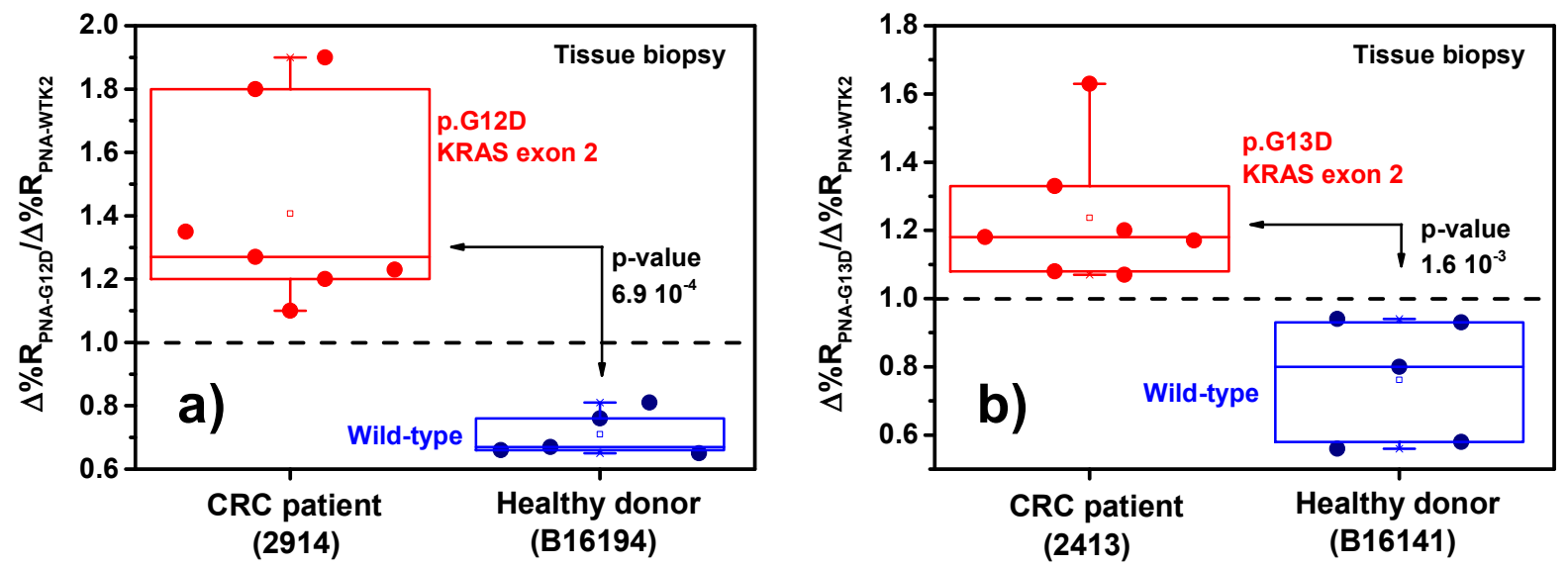

Fig. 6: $\Delta \% \mathrm{R}_{\mathrm{PNA}-M u t} / \Delta \% \mathrm{R}_{\mathrm{PNA} \text {-WTK2}}$ values obtained from replicated NESPRI analysis of tDNA isolated from FFPE samples of CRC patients (samples 2914 and 2413) and gDNA from PBMC of healthy donors (samples B16194 and B16141).

Fig. 6 summarizes results from replicated NESPRI analysis of samples 2914 (p.G12D) and 2413 (p.G13D) from tissue specimens of CRC patients and wild-type specimens from PBMCs of healthy donors (B16194 and B16141 Obtained from Regina Elena Institutional BioBank). Both $\Delta \% \mathrm{R}_{\text {PNA- }}$ Mut $/ \Delta \% R_{\text {PNA-WTK2 }}$ ratios for p.G12D (population mean confidence interval at the $99 \%$ level for $\Delta \% \mathrm{R}_{\text {PNA-G12D }} / \Delta \% \mathrm{R}_{\text {PNA-WTK2 }}$ ratio $\mathrm{CI}=1.40714 \pm 0.439$, replicate analyses $\mathrm{n}=7$ ) and $\mathrm{p} . \mathrm{G} 13 \mathrm{D}$ (population mean confidence interval at the $99 \%$ level for $\Delta \% \mathrm{R}_{\text {PNA-G13D }} / \Delta \% \mathrm{R}_{\text {PNA-WTK2 }}$ ratio $\mathrm{CI}=$ $1.23714 \pm 0.271, \mathrm{n}=7)$ were significantly different than those from healthy donors (B16194 $\Delta \% \mathrm{R}_{\text {PNA-G12D }} / \Delta \% \mathrm{R}_{\text {PNA-WTK2 }} 99 \% \mathrm{CI}=0.71 \pm 0.146, \mathrm{n}=5 . \mathrm{B} 16141 \Delta \% \mathrm{R}_{\mathrm{PNA}-\mathrm{G} 13 \mathrm{D}} / \Delta \% \mathrm{R}_{\mathrm{PNA}-\mathrm{WTK} 2} 99 \%$ $\mathrm{CI}=0.762 \pm 0.379, \mathrm{n}=5)$.

NESPRI directly detects RAS mutated DNA spiked in human plasma. Having demonstrated the analytical capacity of our NESPRI assay on tDNAs, we moved to plasma samples. Plasma is the preferred biological fluid for the analysis of ctDNAs. It is preferred to the serum that is often contaminated with gDNA released by white blood cells as a consequence of lysis occurring during clotting ${ }^{6}$. Nonetheless, plasma obtained from liquid biopsies of cancer patients is a complex matrix 
containing ultra-low concentrated ctDNAs, proteins, non-target nucleic acids and other entities at variable levels ${ }^{22,23}$. Standard protocols for ctDNA analysis from LB include the extraction of ctDNA from the plasma and its PCR-amplification and analysis in buffered solutions ${ }^{24,25}$. New methods capable to directly detect ctDNA in plasma are therefore highly desirable. These would drastically reduce assay time, streamline diagnostic workflows, and minimize any potential risk of sample contamination.

Then we developed a simple one-step pre-analytical procedure (taking 1.5 hours of which 10-12 minutes of hands-on procedures) to digest the large amounts of plasma proteins and make plasma suitable for NESPRI direct analysis ${ }^{26}$. Specifically, we digested plasma samples from healthy donors with proteinase K (ProK $35 \mu$ M. 1:10 diluted with ProK buffer), then we spiked the sample with p.G12D (KRAS exon 2) mutated gDNA isolated from LS174T cells ${ }^{27}$. Spiking was used as a proxy of ctDNA detection in CRC patients.

We immobilized PNA-WTK2, and PNA-G12D probes on the modified gold surface as already described (Supplementary Fig. S2a) and blocked residual reactive NHS ester groups with Trizma (0.1 M, $10 \mathrm{~min})$. The ProK treated and spiked sample was introduced in the microfluidic devices for adsorption on PNA probes after sonication ( $3 \mathrm{~min})$, vortexing $(1 \mathrm{~min})$, heating at $95^{\circ} \mathrm{C}(5 \mathrm{~min})$, and incubation for $1 \mathrm{~min}$ on ice immediately before the analysis. The assay also includes treatment with dithiothreitol (DTT) of the sensor surface to remove any protein non-specifically adsorbed during the surface exposure to plasma (Supplementary Fig. S2b).

Fig. 7 summarizes results from replicated experiments performed with spiked human plasma samples (Supplementary Table S2, Fig. S2c,d). p.G12D gDNA were added to the treated plasma to obtain a final concentration of $2 \mathrm{pg} \mu \mathrm{L}^{-1}(\sim 1 \mathrm{aM})$. We loaded $300 \mu \mathrm{L}$ of the treated plasma sample, carrying a total of about 180 molecules of mutated DNA, for each NESPRI test. Data displayed in Fig. 7 refer to $\Delta \% \mathrm{R}_{\text {PNA-G12D }} / \Delta \% \mathrm{R}_{\text {PNA-WTK2 }}$ ratio of NESPRI signals detected in spiked plasma samples following hybridization with PNA probes complementary to mutated and wild-type 
sequences, respectively, and NPs@MixRAS adsorption. We treated the surface with DTT (100 mM in water, 20 min.) before NPs@MixRAS adsorption.

NESPRI replicated analysis of $2 \mathrm{pg} \mu \mathrm{L}^{-1}$ samples of G12D in human plasma (population mean confidence interval at the $99 \%$ level for $\Delta \% \mathrm{R}_{\text {PNA-G12D }} / \Delta \% \mathrm{R}_{\text {PNA-WTK2 }}$ ratio $\mathrm{CI}=1.20 \pm 0.06$, replicate analyses $\mathrm{n}=13$ ) and wild-type human plasma samples (population mean confidence interval at the $99 \%$ level for $\Delta \% \mathrm{R}_{\text {PNA-G12D }} / \Delta \% \mathrm{R}_{\text {PNA-WTK2 }} \mathrm{CI}=0.84 \pm 0.05$, replicate analyses $\mathrm{n}=10$ ) generated significantly different $\Delta \% \mathrm{R}_{\text {PNA-G12D }} / \Delta \% \mathrm{R}_{\text {PNA-WTK2 }}$ ratios (two-tailed t test, level $99 \%, p$-value $=2.80$ $\left.10^{-10}\right)$.

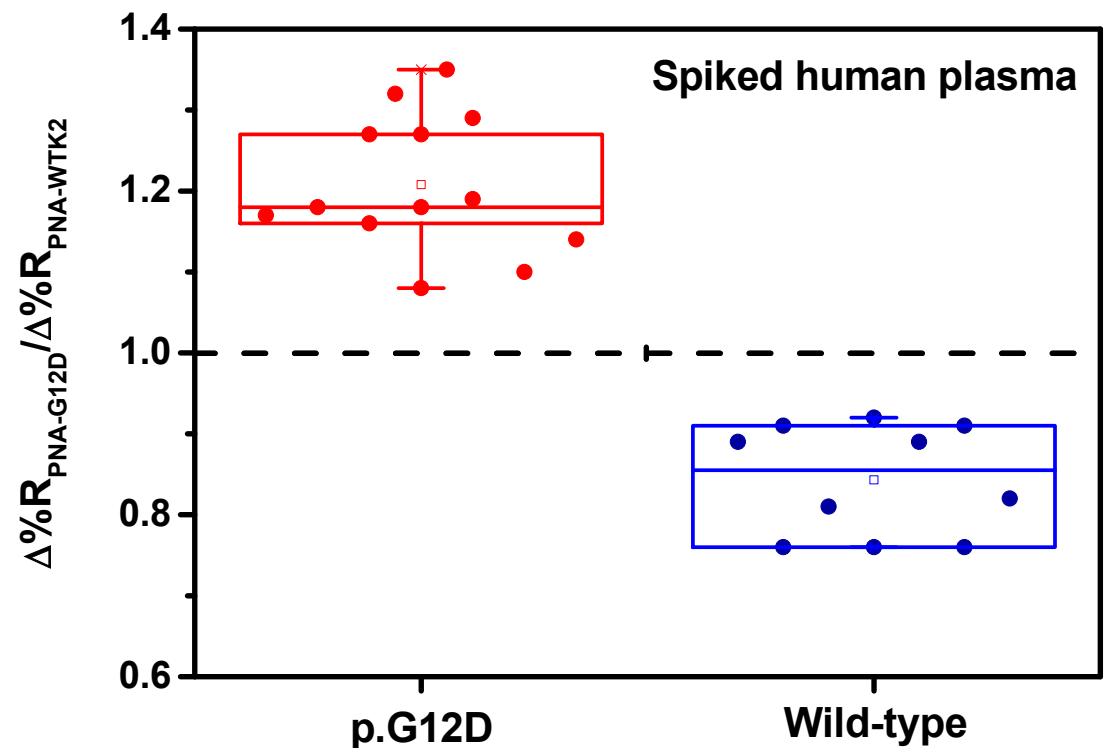

Fig. 7: $\Delta \% \mathrm{R}_{\mathrm{PNA}-\mathrm{G} 12 \mathrm{D}} / \Delta \% \mathrm{R}_{\mathrm{PNA}-\mathrm{WTK} 2}$ values obtained from replicated NESPRI experiments aimed at detecting p.G12D mutated gDNAs spiked in plasma samples from healthy donors and wild-type cfDNA in plasma from healthy donors. p.G12D mutated gDNA isolated from LS174T cells was added to ProK treated human plasma to get the final concentration of $2 \mathrm{pg} \mu \mathrm{L}^{-1} . \Delta \% \mathrm{R}_{\mathrm{PNA}}$ G12D/ $\Delta \%$ RPNA-WTK2 ratio calculated from NESPRI signals detected after the adsorption of NPs@MixRAS on surfaces resulting from the interaction of DNA in plasma samples with PNA probes complementary to the p.G12D mutated and wild-type sequences, respectively.

\section{NESPRI directly detects KRAS mutated ctDNA in liquid biopsy samples from CRC patients.}

Spiking experiments prompted us to apply the NESPRI assay to the direct detection of KRAS mutated ctDNA present in the blood of metastatic CRC patients. These specimens were 
preliminarily validated by NGS and digital PCR $(\mathrm{dPCR})^{28}$, carried a unique $R A S$ mutation per sample and displayed different variant allele frequencies (VAF, i.e., the percentage of mutated reads) and mutated copies per $\mathrm{ml}$ (Table 4$)$.

\begin{tabular}{|c|c|c|c|c|}
\hline \multirow{2}{*}{$\begin{array}{l}\text { Sample } \\
\text { ID }\end{array}$} & \multirow{2}{*}{$\begin{array}{c}\text { KRAS } \\
\text { mutational } \\
\text { status }\end{array}$} & \multicolumn{2}{|c|}{$\begin{array}{l}\text { Circulating DNA in plasma } \\
\left(\text { (copies } \mathrm{mL}^{-1}\right)^{*}\end{array}$} & \multirow{2}{*}{$\begin{array}{c}\text { Mutant } \\
\text { VAF (\%) }\end{array}$} \\
\hline & & ctDNA & WT & \\
\hline $\mathrm{pt} \# 33$ & p.G12V & 1983 & 6438 & 23.5 \\
\hline $\mathrm{pt} \# 34$ & p.G13D & 12215 & 15547 & 44 \\
\hline $\mathrm{pt} \# 40$ & p.G12A & 2617 & 4028 & 39.4 \\
\hline $\mathrm{pt} \# 50$ & p.G12R & 593 & 3412 & 14.8 \\
\hline$\# 2$ & Wild-type & $<1 * *$ & $1473^{\dagger}$ & $<0.01$ \\
\hline$\# 4$ & Wild-type & $<1$ & $1514^{\dagger \dagger}$ & $<0.01$ \\
\hline$\# 8$ & Wild-type & $<1$ & $1602^{\dagger \dagger \dagger}$ & $<0.01$ \\
\hline pool\#4*** & Wild-type & $<1$ & $1534^{\dagger+\dagger \dagger}$ & $<0.01$ \\
\hline $\begin{array}{l}\text { *from dPCR. * } \\
\text { with ( } \dagger \text { KRRAS } \\
\text { p.G12R assay. }\end{array}$ & $\begin{array}{l}\text { t detectable } \\
2 \mathrm{D},(\dagger \dagger) \mathrm{KF}\end{array}$ & $\begin{array}{l}\text { ** Pooled p } \\
\text { S p.G12A, }\end{array}$ & $\begin{array}{l}\text { om health } \\
\text { AS p.G1? }\end{array}$ & $\begin{array}{l}\text { nors. Teste } \\
(\dagger+\dagger) \text { KRA }\end{array}$ \\
\hline
\end{tabular}

We applied the already described NESPRI assay operating under continuous flow conditions so that kinetic curves useful to check the correct outcome of each step of the assay were also recorded during the experiments. 

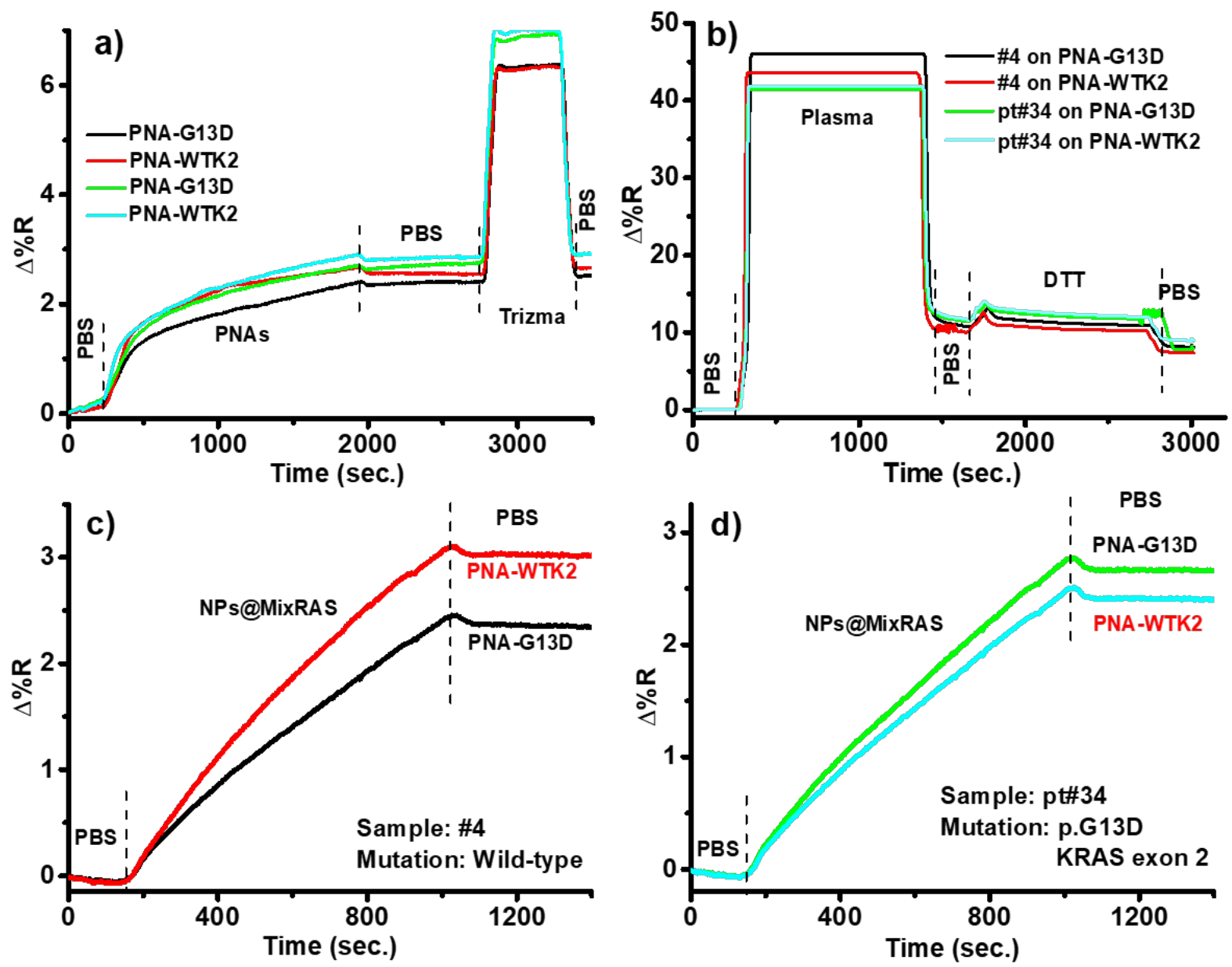

Fig. 8: a) Representative changes in per cent reflectivity $(\Delta \% \mathrm{R})$ over time obtained for the immobilization of PNA-G13D and PNA-WTK2 probes. Curves shown refer to independent immobilizations performed in parallel. b) $\Delta \% \mathrm{R}$ over time detected for the adsorption of treated plasma samples from CRC patient (pt\#34) and healthy donor (\#4) on PNA probes. Each sample $(400 \mu \mathrm{L}$ of 1:10 diluted and treated plasma, flow rate $\left.10 \mu \mathrm{L} \mathrm{min}^{-1}\right)$ was adsorbed on both PNA-G13D and PNA-WTK2 probes. The surface was then treated with DTT (100 mM in water). c,d) $\Delta \% \mathrm{R}$ over time detected for the adsorption of NPs@MixRAS ( $0.1 \mathrm{nM}$ in PBS. Aged $\sim 60$ days) on the resulting surfaces. Plasma samples from (c) a healthy donor (\#4) and (d) a CRC patient with p.G13D mutated ctDNA (pt\#34) produced different signal enhancement when NPs@MixRAS were adsorbed on surfaces with PNA-WTK2 or PNA-G13D.

Representative data for the NESPRI detection of KRAS mutated ctDNA (sample pt\#34), and wildtype cfDNA from a healthy donor (\#4) are shown in Fig. 8. PNA-WTK2 and PNA-G13D probes were immobilized using a microfluidic device bearing parallel microchannels (Fig. 8a) for the parallel analysis of both plasma samples treated as already described. After the adsorption of $400 \mu \mathrm{L}$ of each 1:10 diluted and treated plasma sample on both PNA-WTK2 and PNA-G13D and the 
exposure of the resulting surfaces to DTT (Fig. 8b) we used NPs@MixRAS to get the final detection (assay time about 80 minutes). Fig. 8c shows NESPRI responses for the wild-type cfDNA from healthy donors (sample \#4), whereas Fig. 8d refers to the plasma of CRC patient (pt\#34). The detected NESPRI responses demonstrate the preferential interaction p.G13D mutated ctDNA with PNA-G13D and the preferential interaction of wild-type cfDNA with PNA-WTK2.
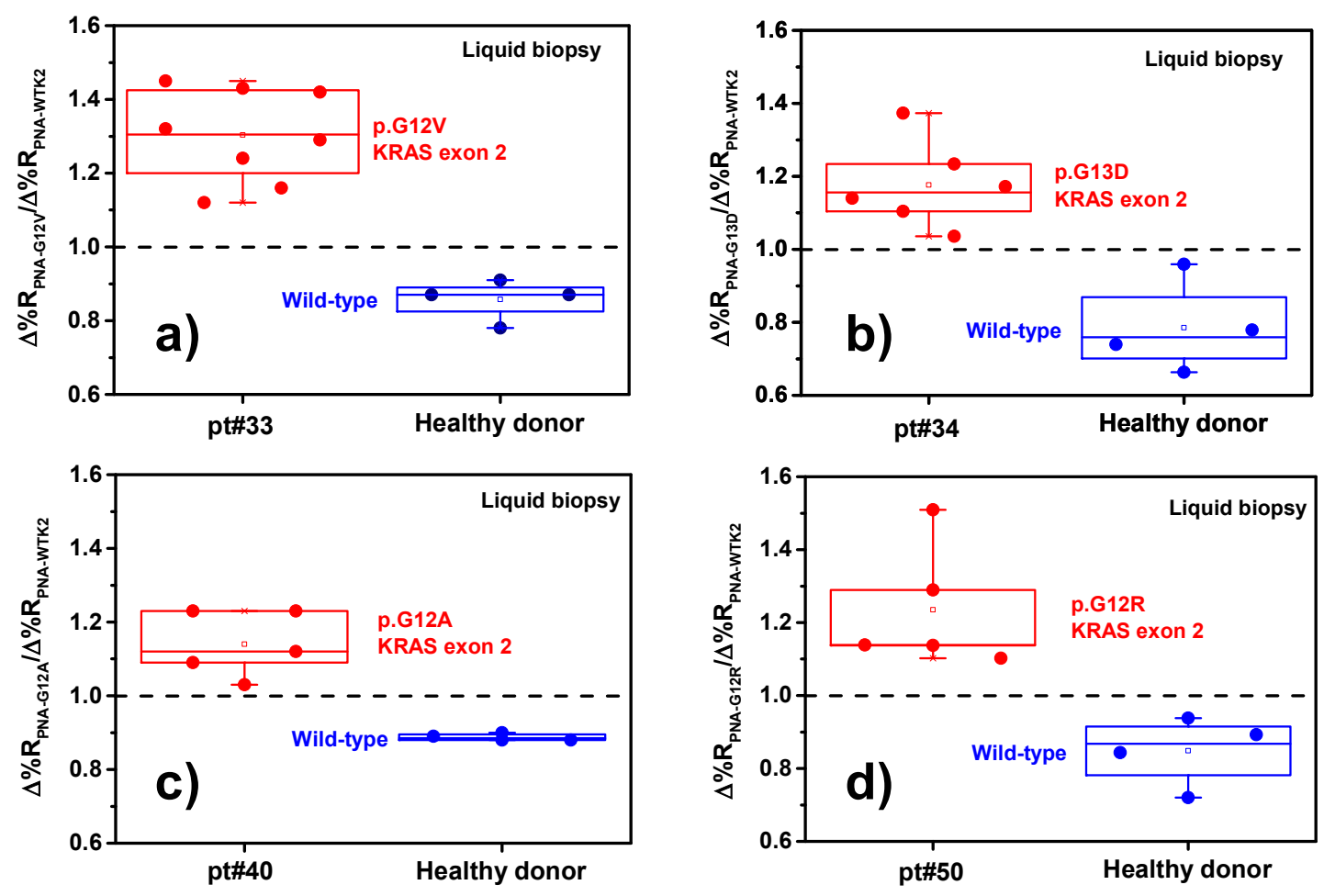

Fig. 9: Box plots of the $\Delta \% \mathrm{R}_{\mathrm{PNA}-\mathrm{Mut}} / \Delta \% \mathrm{R}_{\mathrm{PNA}-\mathrm{wTK} 2}$ ratio calculated after the adsorption of NPs@MixRAS. Plasma samples from CRC patients with $R A S$ mutated ctDNAs provide values of the ratio greater than 1 , whereas plasma samples from healthy donors (wild-type cfDNA) provide values smaller than 1 .

Fig. 9 summarizes results $\left(\Delta \% \mathrm{R}_{\mathrm{PNA}-\mathrm{Mut}} / \Delta \% \mathrm{R}_{\mathrm{PNA} \text {-WTK} 2}\right)$ obtained from replicated analyses of liquid biopsies from $R A S$ mutated CRC patients and healthy donors that we analyzed in parallel to assess the capacity of the NESPRI assay to discriminate among the two groups. Population mean confidence intervals of $\Delta \% \mathrm{R}_{\text {PNA-Mut }} \Delta \% \mathrm{R}_{\text {PNA-WTK2 }}$ ratios for the analyzed liquid biopsies are reported in Table 5, together with ID of samples used for the analysis, the number of replicates and p-values (CRC patient and healthy donor mean comparison. Two-tailed t-test). 


\begin{tabular}{|c|c|c|c|}
\hline Sample ID & $\begin{array}{c}\text { Population mean } \\
\text { confidence interval } \\
\Delta \% R_{\text {PNA-Mut } / \Delta \% R_{\text {PNA-WTK2 }}} \\
\text { (level } 99 \%)\end{array}$ & Replicates & p-value \\
\hline $\mathrm{pt} \# 33$ & $1.30 \pm 0.15$ & 8 & \multirow{2}{*}{$5.510^{-5}$} \\
\hline$\# 2, \# 8$ & $0.86 \pm 0.16$ & 4 & \\
\hline $\mathrm{pt} \# 34$ & $1.18 \pm 0.19$ & 6 & \multirow[t]{2}{*}{$9.910^{-4}$} \\
\hline$\# 4, \# 8$ & $0.78 \pm 0.37$ & 4 & \\
\hline $\mathrm{pt} \# 40$ & $1.14 \pm 0.18$ & 5 & \multirow[t]{2}{*}{$8.010^{-4}$} \\
\hline pool\#4 & $0.89 \pm 0.03$ & 4 & \\
\hline $\mathrm{pt} \# 50$ & $1.23 \pm 0.35$ & 5 & \multirow{2}{*}{$4.810^{-3}$} \\
\hline pool\#4 & $0.85 \pm 0.27$ & 4 & \\
\hline
\end{tabular}

\section{Discussion}

This paper demonstrates that plasmonic-based NESPRI specifically detects ctDNA in the blood of CRC patients. The new method introduces significant improvements as compared to state of the art. The NESPRI assay involves limited sample manipulation and no circulating DNA isolation from plasma before the analysis. Both features help in minimizing the risk of sample contamination and contribute to reducing the assay time. The assay does not require the amplification of the target sequence, thus preventing biases and artefacts introduced by the PCR amplification widely used today to analyze liquid biopsies ${ }^{29,30}$.

Whereas commercial NGS and dPCR equipment can only accept purified and concentrated analyte as input, NESPRI operates with human plasma that has undergone minimal pre-analytical manipulation, e.g. addition of ProK. Therefore, NESPRI is likely to greatly simplify and speed up the tedious (although automatable) pre-analytical process of ctDNA extraction and concentration. The simplified NESPRI pre-analytical processing takes 1.5 hours (of which only 10-12 minutes of hands-on procedures), which is 2-3 times shorter and far less error-prone than the extensive sample 
handling necessary for the pre-analytical treatment of human plasma upstream to dPCR and/or NGS. Remarkably, this favorable feature is likely to remain unique of NESPRI. Even assuming that laboratory equipment might be adapted to accept more diluted analytes, NGS and APCR reaction mixes would still contain both ProK and DNA amplification/sequencing enzymes (typically Taq polymerase), resulting in digestion and inactivation of the latter. In contrast, NESPRI performs an enzyme-free detection based on a sandwich assay configuration, which permits to run the nanoparticle-supported signal enhancement after suitable surface treatments.

In addition, and also importantly, NESPRI operates with volumes as low as $40 \mu \mathrm{L}$ of human plasma, whereas NGS and APCR require a minimal DNA input of a few hundreds of microliters of plasma, e.g. at least one order of magnitude more blood than NESPRI. This difference, however, is likely to be underestimated, because effective analyte recovery/handling (e.g. from ion exchange microcolumns and/or magnetic devices) for NGS/dPCR usually requires the processing of a few millilitres of plasma, e.g. an amount in excess to that needed for a single NGS/dPCR run. Thus, a significant fraction of the blood draw is irreversibly manipulated, and cannot be made available for other OMIC assessments except those compatible with the intended pre-analytical procedure.

In contrast, NESPRI leaves any unused aliquot of plasma untouched for both intended and, most importantly, unanticipated uses. Thus, NESPRI takes advantage of a non-destructive, fully scalable, ultra-low volume pre-analytical processing which makes it particularly suitable not only for quick turnaround times in real-life oncology, but also for scalable, fully customizable LB testing, thus enabling truly adaptive clinical trial schemes.

The exceptional stability of PNA/DNA duplexes is another key feature of the NESPRI assay ${ }^{31}$. Biological stability of PNAs is also excellent since they are not degraded by enzymes occurring in biological samples ${ }^{32}$. PNA probes were also selected for their ability to discriminate single-base mismatches compared with standard oligonucleotide probes $^{33}$, thus enhancing the capacity of the sensor to distinguish between mutated and wild-type circulating DNAs. This ability is enhanced 
when working either at high temperatures or high dilutions ${ }^{34}$, the latter being ideal for detection of very low DNA quantities, as in the present study.

The enhancement of the plasmonic signal is, however, necessary for the read-out of the differential interactions occurring between PNA probes and target ctDNA. We achieved the required enhancement using gold nanoparticles (NPs@MixRAS). In particular, we detected ctDNAs comparing the NPs@MixRAS-enhanced responses after the parallel exposure of PNA-Mut and PNA-WT probes to the selected liquid biopsy. With this approach, the effects of non-specific adsorption involving both probes in an almost identical manner are compensated, and only the ratio of signals generated by the specific interaction of the sample with PNA-Mut and PNA-WT probes differing at a single base is evaluated. The described approach implies an appropriate control of the surface coverage of the immobilized probes (about $3 \times 10^{12}$ molecules $\mathrm{cm}^{-2}$ ) that in our case produced a signal variability typically ranging from $\mathrm{CV}=3 \%$ and $\mathrm{CV}=7 \%$ for the six parallel performed immobilizations. PNA probes immobilizations with $\mathrm{CV} \geq 10 \%$ were discarded.

An essential aspect of the NESPRI assay is represented by its simplicity and potential for multiplexed detection. In this light, we demonstrated that the use of NPs@MixRAS allows NESPRI detection of 11 among the most frequent ALL-RAS cancer mutations (Table 2).

The analysis of the ROC curve referring to NESPRI detection of tDNA isolated from tissue biopsies of CRC patient afforded an AUC of 0.947 with $100 \%$ sensitivity and $83.33 \%$ specificity (Fig. 5 . Table 3). These results indicate that our NESPRI method detects mutated DNA from tissue biopsy accurately. Spiking plasma with p.G12D gDNA attained an analytical sensitivity of $2 \mathrm{pg} \mu \mathrm{L}^{-1}$, sufficiently low to meet most clinical needs ${ }^{35}$.

The direct adsorption of the spiked plasma samples on NESPRI surface results in non-specific adsorption of interfering species present in the biological sample at the functional interface of the biosensor. In our case, treatment of the surface with DTT restored the typical NPs@MixRASenhanced response allowing the detection of $\sim 1$ aM p.G12D mutated DNA and discrimination between p.G12D and wild-type samples (Fig. 7). 
The ctDNA status has been repeatedly found to correlate with, and possibly surrogate for, the $R A S$ mutational status in tissue biopsies from CRC patients ${ }^{36,37}$. However, detection of KRAS mutated ctDNA with no correspondence with the mutational status of tissue biopsy has also been repeatedly reported ${ }^{38}$, expanding the diagnostic implications of $\mathrm{LB}^{39}$.

Data here reported displays the clinical validity of the NESPRI assay and its ability to discriminate between normal and $R A S$ mutated genotype into separate groups (CRC and control group). These

were shown to be associated with different treatment indications and clinical outcomes ${ }^{28}$. The assay has been shown to exhibit analytical validity also in testing liquid biopsies from CRC patients. NESPRI is not subject to several constraints affecting methods currently applied in the clinical practice for cancer diagnosis, prognosis and patient follow-up. Current methods require multiple primers for each specific type of mutant ${ }^{40}$, ctDNA or cfDNA to be amplified by PCR, complex sample processing and labelling and an input volume of the human plasma that sometimes introduces a significative constraint for the application of the liquid biopsy approach to real life diagnostics ${ }^{41}$. NESPRI assay leads to a much simpler assay workflow compared to currently available technologies. It minimizes the risk of sample contamination and sample loss and significantly reduces the operative time for pre-analytical procedures. Finally, NESPRI multiplex analysis capability deriving from its imaging setup and microfluidic management of samples will allow straightforward automation of the approach and a low cost per test.

\section{Methods}

\section{Cell lines and human specimens}

Human CRC cell lines HT-29 (All-RAS WT) and LS147T (KRAS p.G12D) genomic DNA isolation protocols are elsewhere described ${ }^{27}$. Blood from healthy donors was obtained from Regina Elena Institutional BioBank. Anonymized clinical samples were donated by stage IV CRC patients and healthy blood donors, as described ${ }^{28}$. The study was approved by the IRCCS Regina Elena National Cancer Institute Ethical Review Board (authorization ID \#CEC/541/15). Briefly, 
conventional histological sections $(5 \mu \mathrm{m}$-thick) from a representative formalin-fixed, paraffinembedded (FFPE) tumor tissue block were stained and assessed by an expert pathologist for a tumor fraction $\geq 70 \%$. Tissue DNA was extracted from deparaffinized sections by the QIAmp DNA FFPE Tissue Kit (Qiagen) according to the manufacturer's instructions. Blood (20 ml) was drawn in BD Vacutainer K2EDTA tubes. Plasma and cfDNA were obtained, handled, processed and purified as described ${ }^{28}$, the latter using the QIAmp circulating nucleic acid kit (Qiagen). All gDNAs and cfDNAs were KRAS genotyped by targeted NGS: the former with with the 50-gene Ion AmpliSeq ${ }^{\mathrm{TM}}$ Cancer Hotspot Panel v2; the latter with the 52-gene Oncomine Pan-Cancer Cell-Free panel (both from Thermofisher Scientific), as per manufacturer's instructions. KRAS genotyping was confirmed by specific digital PCR (dPCR) assays using as templates either gDNA (20 ng) or ctDNA (from $0.5 \mathrm{~mL}$ of plasma) in the chip-based QuantStudio ${ }^{\mathrm{TM}}$ 3D Digital PCR System (Life Technologies). 2D plots (WT/MUT) were automatically elaborated using the on-board Thermo Fisher Cloud Analysis Suite Variant. Allelic Frequencies (VAF) were similarly (+/- 1.5\%) estimated by NGS and dPCR. The complete list of indexed and custom-designed dPCR assays is presented in Supplementary Table S3.

\section{Design and synthesis of PNA probes for $R A S$ mutations}

The PNA sequence selection was made according to the above mentioned Tm predictions, and evaluation of possible interferent by BLAST search. The presence of self-paired sequences was also minimized since these are known to prevent interaction with complementary DNA. Two 2-(2aminoethoxy)ethoxyacetic acid (AEEA) linkers were added at the $\mathrm{N}$ terminus of PNA probes to increase the accessibility of the target DNA, according to our previous studies using microarray technology ${ }^{42}$. All PNAs were produced using automatic solid-phase synthesis with standardized protocols ${ }^{43}$, purified by HPLC and characterized by UPLC-MS, as described previously ${ }^{44}$, for purity and identity assessment (see Supplementary Figures S3-S17 for full characterization).

\section{NESPRI measurements}


We performed NESPRI experiments at room temperature and using an SPR imager apparatus (GWC Technologies, U.S.A.) and analyzed the detected images by using V++ software (version 4.0, Digital Optics Limited, New Zealand) and the ImageJ 1.32j software package (National Institutes of Health, U.S.A.). SPRI image data (pixel intensity, 0-255 scale) were converted into percentage of reflectivity $(\% \mathrm{R})$ using the equation $\% \mathrm{R}=100 \times 0.85 \mathrm{I}_{\mathrm{p}} / \mathrm{I}_{\mathrm{s}}$ where Ip and Is refer to the reflected light intensity detected using p- and s-polarized light, respectively. Experiments were carried out by sequentially acquiring 15 frames averaged SPR images with $5 \mathrm{~s}$ time delay between them. We obtained kinetic data plotting the difference in $\% \mathrm{R}(\Delta \% \mathrm{R})$ from selected regions of interest of SPR images as a function of time. A microfluidic device with six parallel microchannels (80 $\mu \mathrm{m}$ depth, $1.4 \mathrm{~cm}$ length, $400 \mu \mathrm{m}$ width) was used for the study, to allow independent control of interactions occurring on six different regions of the NESPRI gold chip surface. The device was fabricated in poly(dimethylsiloxane) (PDMS) polymer by replica molding ${ }^{45}$. PEEK tubes (UpChurch Scientific) were inserted into the microfluidic device to connect the microfluidic cell to an Ismatec IPC (Ismatec SA, Switzerland) peristaltic pump. A refractive index matching liquid was used to obtain the optical contact between the gold chip and the prism.

\section{Gold surface modification}

Gold chips (Xantec Bioanalytics, Germany) functionalized with DTSP, were used to immobilize PNA-WT and PNA-Mut probes. We obtained DTSP functionalization as follows: a bare gold chip was treated with UV/ozone for $5 \mathrm{~min}$, then washed with ethanol for $10 \mathrm{~min}$ and dried under a nitrogen stream. The cleaned chip was immersed for $48 \mathrm{~h}$ in DTSP solution (4 $\mathrm{mM}$ in DMSO) under constant and gentle agitation at $23^{\circ} \mathrm{C}$. The modified chip was then thoroughly rinsed with ethanol. PNA-WT and PNA-Mut probes were immobilized on DTSP-modified gold chips through amine-coupling reaction between N-hydroxysuccinimidyl (NHS) ester ends of DTSP and the Nterminal group of (AEEA) 2 linker. The spatially separated immobilization of PNA-WT and PNAMut probes was obtained by injecting PNA-WT or PNA-Mut solutions $(0.1 \mu \mathrm{M}$ in PBS, flow rate 
$10 \mu \mathrm{L} \mathrm{min}{ }^{-1}$ ) into the parallel channels of the microfluidic device in contact with the DTSPmodified gold surface.

\section{Nanoparticles synthesis and functionalization}

We obtained AuNPs using the citrate reduction of tetrachloroauric acid method elsewhere described $^{46}$. We used Milli-Q purified water and analytical grade reagents. Glassware was cleaned with aqua regia $\left(3: 1\right.$ mixture of concentrated $\mathrm{HCl}$ and $\left.\mathrm{HNO}_{3}\right)$ and piranha solution $(3: 1$ mixture of concentrated $\mathrm{H}_{2} \mathrm{SO}_{4}$ and $30 \% \mathrm{H}_{2} \mathrm{O}_{2}$ ), rinsed with water and dried under a stream of nitrogen to avoid unwanted nucleation during the synthesis, as well as aggregation of gold colloids.

The procedure provided monodispersed spherical AuNPs with an average diameter of $15 \mathrm{~nm}$ and a surface area availability per nanoparticle of about $625 \mathrm{~nm}^{2}$. Properties of dispersed nanoparticles were also determined by optical extinction spectroscopy, dynamic light scattering (DLS) and $\zeta$ potential (Supplementary Table S5)

We obtained functionalized AuNPs with high colloidal stability and facile chemical functionality by passivation with polyvinylpyrrolidone (PVP) polymer. PVP is an amphiphilic polymer bearing a hydrophobic chain and highly polar pyrrolidone side groups. After adsorption of PVP on AuNPs surface, highly polar side groups are densely exposed at the surface of the modified nanoparticles (NPs@PVP). The passivation provides excellent colloidal stability. Modified AuNPs are stabilized in PBS up to more than 14 months. Streptavidin (SA) was then adsorbed on NPs@PVP and biotinylated oligonucleotides immobilized on NPs@PVPSA. We selected oligonucleotide to be immobilized on NPs@PVPSA by identifying sequences that, in combination with PNA probe sequence, have less mutually interfering sequences in the human genome. NP@MixRAS were obtained by immobilizing three different oligonucleotides (Table 2) on NPs@PVPSA and using the protocol described in detail in Supplementary information. (Supplementary Section S2).

Optical absorption spectra of AuNPs dispersions before and after the functionalization with the selected oligonucleotide are shown in Supplementary Fig. S18 and Table S6.

\section{Plasma samples processing protocol}


Wild-type or mutated human plasma samples, 1:10 diluted with Proteinase K buffer (20 mM Tris $\mathrm{HCl}, 1 \mathrm{mM} \mathrm{CaCl} 2,50 \%$ glycerol, $\mathrm{pH} 8$ ), were treated by incubating the unprocessed samples or p.G12D spike-in samples, with $35 \mu \mathrm{M}$ ProK $(20 \mu \mathrm{L}$ in $400 \mu \mathrm{L}$ ProK at $20 \mathrm{mg} / \mathrm{mL})$ for $1.5 \mathrm{~h}$ at $37^{\circ} \mathrm{C}$ under gentle agitation. We used human plasma centrifuged at $16000 \mathrm{~g}$ for 20 minutes at $4^{\circ} \mathrm{C}$. Before NESPRI analyses, gDNA or cfDNA and ctDNA in plasma samples were fragmented by sonication (3 min, ELMA Transsonic T480/H-2) and vortexing (1 min, IKA Vortex GENIUS 3) and denatured by heating at $95{ }^{\circ} \mathrm{C}$ for $5 \mathrm{~min}$. Strands re-association was prevented by cooling on ice samples (1 min) before their introduction into NESPRI microfluidics apparatus. 


\section{References}

1. Heitzer, E., Roberts, C. E. S. \& Speicher, M. R. Current and future perspectives of liquid biopsies in genomics-driven oncology. Nat. Rev. Genet. 20, 71-88 (2019).

2. Crowley, E., Di Nicolantonio, F., Loupakis, F. \& Bardelli, A. Liquid biopsy: monitoring cancer-genetics in the blood. Nat. Rev. Clin. Oncol. 10, 472-484 (2013).

3. Eisenstein, M. Taking cancer out of circulation. Nature 579, S6-S8 (2020).

4. Alix-Panabières, C. \& Pantel, K. Clinical applications of circulating tumor cells and circulating tumor DNA as liquid biopsy. Cancer Discov. 6, 479-491 (2016).

5. Wan, J. C. M. et al. Liquid biopsies come of age: Towards implementation of circulating tumour DNA. Nat. Rev. Cancer 17, 223-238 (2017).

6. Merker, J. D. et al. Circulating tumor DNA analysis in patients with cancer: American society of clinical oncology and college of American pathologists joint review. J. Clin. Oncol. 36, 1631-1641 (2018).

7. Bardelli, A. \& Pantel, K. Liquid Biopsies, What We Do Not Know (Yet). Cancer Cell 31, $172-179$ (2017).

8. Siravegna, G., Marsoni, S., Siena, S. \& Bardelli, A. Integrating liquid biopsies into the management of cancer. Nat. Rev. Clin. Oncol. 14, 531-548 (2017).

9. Volckmar, A. L. et al. A field guide for cancer diagnostics using cell-free DNA: From principles to practice and clinical applications. Genes Chromosom. Cancer 57, 123-139 (2018).

10. Chen, M. \& Zhao, H. Next-generation sequencing in liquid biopsy: cancer screening and 
early detection. Hum. Genomics 13, 34 (2019).

11. Zehir, A. et al. Mutational landscape of metastatic cancer revealed from prospective clinical sequencing of 10,000 patients. Nat. Med. 23, 703-713 (2017).

12. Goodwin, S., McPherson, J. D. \& McCombie, W. R. Coming of age: Ten years of nextgeneration sequencing technologies. Nat. Rev. Genet. 17, 333-351 (2016).

13. Hu, Y. et al. False-positive plasma genotyping due to clonal hematopoiesis. Clin. Cancer Res. 24, 4437-4443 (2018).

14. Spoto, G. \& Corradini, R. Detection of Non-Amplified Genomic DNA. (2012). doi:10.1007/978-94-007-1226-3

15. Vynck, M. \& Thas, O. Reducing Bias in Digital PCR Quantification Experiments: The Importance of Appropriately Modeling Volume Variability. Anal. Chem. 90, 6540-6547 (2018).

16. Bocková, M., Slabý, J., Špringer, T. \& Homola, J. Advances in Surface Plasmon Resonance Imaging and Microscopy and Their Biological Applications. Annu. Rev. Anal. Chem. 12, $151-176(2019)$.

17. D’Agata, R., Giuffrida, M. C. \& Spoto, G. Peptide Nucleic Acid-Based Biosensors for Cancer Diagnosis. Molecules 22, 1-15 (2017).

18. D’Agata, R., Palladino, P. \& Spoto, G. Streptavidin-coated gold nanoparticles: Critical role of oligonucleotides on stability and fractal aggregation. Beilstein J. Nanotechnol. 8, 1-11 (2017).

19. D'Agata, R. et al. Direct detection of point mutations in nonamplified human genomic DNA. Anal. Chem. 83, 8711-8717 (2011). 
20. Mullard, A. Cracking KRAS. Nat. Rev. Drug Discov. 18, 887-891 (2019).

21. PNA:DNA (with full match complementary DNA) melting temperatures were calculated for $4 \mu \mathrm{M}$ concentration, using the on-line available PNA design tool from PNA-BIO (http://pnabio.com/support/PNA_Tool.htm), according to: Giesen, U. et al. A formula for thermal stability $(\mathrm{T}(\mathrm{m}))$ prediction of PNA/DNA duplexes. Nucleic Acids Res. 26, 5004$5006(1998)$.

22. Bellassai, N. \& Spoto, G. Biosensors for liquid biopsy: circulating nucleic acids to diagnose and treat cancer. Anal. Bioanal. Chem. 408, 7255-7264 (2016).

23. Bellassai, N., D’Agata, R., Jungbluth, V. \& Spoto, G. Surface Plasmon Resonance for Biomarker Detection: Advances in Non-invasive Cancer Diagnosis. Front. Chem. 7, 1-16 (2019).

24. Peters, D. L. \& Pretorius, P. J. Origin, translocation and destination of extracellular occurring DNA - A new paradigm in genetic behaviour. Clin. Chim. Acta 412, 806-811 (2011).

25. Jen, J., Wu, L. \& Sidransky, D. An Overview on the Isolation and Analysis of Circulating Tumor DNA in Plasma and Serum. Ann. N. Y. Acad. Sci. 906, 8-12 (2006).

26. Diehl, F. et al. Detection and quantification of mutations in the plasma of patients with colorectal tumors. Proc. Natl. Acad. Sci. U. S. A. 102, 16368-16373 (2005).

27. Gasparello, J. et al. Liquid biopsy in mice bearing colorectal carcinoma xenografts: Gateways regulating the levels of circulating tumor DNA (ctDNA) and miRNA (ctmiRNA). J. Exp. Clin. Cancer Res. 37, 1-11 (2018).

28. Allegretti, M. et al. Cross-sectional analysis of circulating tumor DNA in primary colorectal cancer at surgery and during post-surgery follow-up by liquid biopsy. J. Exp. Clin. Cancer Res. 39, 69 (2020). 
29. Kanagawa, T. Bias and Artifacts in Multitemplate Polymerase Chain Reactions (PCR). J. Biosci. Bioeng. 96, 317-323 (2003).

30. Aird Daniel et al. Analyzing and minimizing PCR amplification bias in Illumina sequencing libraries. Genome Biol. 12, 1-14 (2011).

31. Nielsen, P. E. Peptide Nucleic Acids (PNA) in chemical biology and drug discovery. Chem. Biodivers. 7, 786-804 (2010).

32. Demidov, V. V. et al. Stability of peptide nucleic acids in human serum and cellular extracts. Biochem. Pharmacol. 48, 1310-1313 (1994).

33. Egholm, M. et al. PNA watson-crick. Nature 365, 566-568 (1993).

34. A. Bertucci, A. Manicardi, R. C. Advanced Molecular Probes for Sequence-Specific DNA Recognition, In Detection of non-amplified Genomic DNA. (2012). doi:10.1007/978-94-007$1226-3$

35. Xue, X., Zhu, Y. M. \& Woll, P. J. Circulating DNA and lung cancer. Ann. N. Y. Acad. Sci. 1075, 154-164 (2006).

36. Mouliere, F. et al. Circulating cell-free DNA from colorectal cancer patients may reveal high KRAS or BRAF mutation load. Transl. Oncol. 6, 319-328 (2013).

37. Bettegowda, C. et al. Detection of circulating tumor DNA in early- and late-stage human malignancies. Sci. Transl. Med. 6, (2014).

38. Siravegna, G. et al. Clonal evolution and resistance to EGFR blockade in the blood of colorectal cancer patients. Nat. Med. 21, 795-801 (2015).

39. Normanno, N., Cervantes, A., Ciardiello, F., De Luca, A. \& Pinto, C. The liquid biopsy in the management of colorectal cancer patients: Current applications and future scenarios. 
Cancer Treat. Rev. 70, 1-8 (2018).

40. Mouliere, F., El Messaoudi, S., Pang, D., Dritschilo, A. \& Thierry, A. R. Multi-marker analysis of circulating cell-free DNA toward personalized medicine for colorectal cancer. Mol. Oncol. 8, 927-941 (2014).

41. Li, M., Diehl, F., Dressman, D., Vogelstein, B. \& Kinzler, K. W. BEAMing up for detection and quantification of rare sequence variants. Nat. Methods 3, 95-97 (2006).

42. Germini, A. et al. Development of a peptide nucleic acid array platform for the detection of genetically modified organisms in food. J. Agric. Food Chem. 53, 3958-3962 (2005).

43. Alex Manicardi, Roberto Gambari, Luisa de Cola, and R. C. in 3, 73-102 (2001).

44. Mejia-Ariza, R. et al. DNA Detection by Flow Cytometry using PNA-Modified MetalOrganic Framework Particles. Chem. - A Eur. J. 23, 4180-4186 (2017).

45. Grasso, G., D’Agata, R., Zanoli, L. \& Spoto, G. Microfluidic networks for surface plasmon resonance imaging real-time kinetics experiments. Microchem. J. 93, 82-86 (2009).

46. Turkevich, J., Stevenson, P. C. \& Hillier, J. A study of the nucleation and growth processes in the synthesis of colloidal gold. Discuss. Faraday Soc. 11, 55-75 (1951).

\section{Acknowledgements}

This work was supported by European Union's Horizon 2020 research and innovation programme under grant agreements n. 633937, project ULTRAPLACAD and n.764281, project AiPBAND. Associazione Italiana per la Ricerca sul Cancro (AIRC) is thanked for Nuvenia Fellowship (id 19503 to M.A. and id 19052 to P.G.). We thank Davide Sbravati for assistance in PNA synthesis and characterization. This work has benefited from the equipment and framework of the COMPHUB Initiative, funded by the 'Departments of Excellence' program of the Italian Ministry for Education, University and Research (MIUR, 2018-2022). 


\section{Author contributions}

R.D. and G.S. conceived the idea. R.D. and N.B. performed NESPRI experiments. R.D. synthesized and functionalized nanoparticles. R.C. supervised the production and characterization of PNA probes and designed the experiments. A.M. designed the sequences and established protocols for PNA synthesis. A.R. and S.K. performed the synthesis and characterization of PNAs. A.R. performed melting studies. M.A. performed NGS and dPCR. E.M. performed samples and data collection and annotation. E.P. performed pathological revision and BioBanking. P.G. contributed to conceptualisation and helped writing the manuscript. R.D. and G.S. composed the manuscript with the comments from all authors. G.S. coordinated the study.

\section{Competing interests}

The authors declare no competing interests.

\section{Additional information}

Supplementary information is available for this paper.

Correspondence and requests for materials should be addressed to G.S. 